\section{Accuracy of referrals for visual assessment in a stroke population}

FJ Rowe ${ }^{1}$ and VIS Group ${ }^{2,3}$

\begin{abstract}
Purpose To evaluate accuracy of referrals from multidisciplinary stroke teams requesting visual assessments. Patients and methods Multicentre prospective study undertaken in 20 acute Trust hospitals. Stroke survivors referred with suspected visual difficulty were recruited. Standardised screening/referral and investigation forms were used to document data on referral signs and symptoms, plus type and extent of visual impairment.

Results Referrals for $\mathbf{7 9 9}$ patients were reviewed: $60 \%$ men, $40 \%$ women. Mean age at onset of stroke was 69 years (SD 14: range 1-94 years). Signs recorded by referring staff were nil in $58 \%$ and positive in the remainder.

Symptoms were recorded in $87 \%$. Diagnosis of visual impairment was nil in $8 \%$ and positive in the remainder. Sensitivity of referrals (on the basis of signs detected) was calculated as $\mathbf{0 . 4 2}$ with specificity of $\mathbf{0 . 5 2}$. Kappa statistical evaluation of agreement between referral and diagnosis of visual impairment was 0.428 (SE 0.017: $95 \%$ confidence interval of $-0.048,0.019)$. Conclusion More than half of patient referrals were made despite no signs of visual difficulty being recorded by the referring staff. Visual impairment of varying severity was diagnosed in $92 \%$ of stroke survivors referred for visual assessment. Referrals were made based predominantly on visual symptoms and because of formal orthoptic liaison in Trusts involved. Eye (2011) 25, 161-167; doi:10.1038/eye.2010.173; published online 3 December 2010
\end{abstract}

Keywords: visual impairment; stroke; referral; accuracy; detection

\section{Introduction}

Visual impairment is a common occurrence following stroke and can have considerable impact on general rehabilitation. ${ }^{1-4}$ In many units, the stroke multidisciplinary team includes an Orthoptist, and hence there exists an ease of access to eye services. ${ }^{5}$ In other units, there is no formal eye service link for stroke survivors.

Referral of stroke survivors for additional specialist assessment is generally recommended. ${ }^{6,7}$ It is unknown, however, what referrals for visual assessment encompass, and it is possible that stroke survivors are seen by quite different eye care professionals, depending on the type of referral and the unit in which they are seen. It is also unknown what the accuracy of referrals is from the multidisciplinary team. Thus, the purpose of this study is to evaluate accuracy of referrals from multidisciplinary stroke teams requesting visual assessments.

\section{Materials and methods}

The VIS study has been described in detail elsewhere. ${ }^{1}$ The study is a prospective multicentre observational case-cohort study, which conforms to the Tenets of Helsinki, with multicentre ethical approval in the UK. The VIS group consists of local investigators responsible for assessing stroke patients and collecting patient data. The data is collected centrally at the University of Liverpool. The study commenced in May 2006 with 8 centres, and a further 12 centres joined the study over the ensuing 18 months. All centres volunteered to take part by their own volition. These centres consisted stroke units and populations of varying size. Thus, there were considerable differences in the numbers of patients recruited from each participating centre over the duration of the recruitment period.

The target population is stroke patients suspected of having a visual difficulty. Importantly, the target population could not
${ }^{1}$ Directorate of Orthoptics and Vision Science, University of Liverpool, Liverpool, UK

${ }^{2}$ Multi-centre UK recruiting centres

Correspondence: FJ Rowe, Directorate of Orthoptics and Vision Science, Thompson Yates Building, University of Liverpool, Brownlow Hill, Liverpool L69 3GB, UK.

Tel: + $44(0) 151794$ 5732;

Fax: + $44(0) 1517945781$

E-mail: rowef@

liverpool.ac.uk

${ }^{3}$ Members of the VIS group are listed in the appendix.

Received: 13 May 2010 Accepted in revised form: 21 September 2010; Published online: 3 December 2010 
PATIENT DETAILS

Name:

Address:

Telephone:

Hospital number:

DETAILS OF STROKE

Date of onset:
PRIORITY*

Soon Urgent

Current ward / department:

Male / female:

$\mathrm{MRI} / \mathrm{CT}$ date and report:

OCULAR SYMPTOMS Does the patient complain of:

$\begin{array}{lllll}\text { Diplopia } & \begin{array}{l}\text { Blurred/reduced } \\ \text { vision }\end{array} & \begin{array}{l}\text { Reading } \\ \text { difficulties }\end{array} & \begin{array}{l}\text { Visual field loss } \\ \text { or inattention }\end{array} & \text { Other (specify) }\end{array}$

OCULAR SIGNS Are any of the following evident:

\begin{tabular}{|c|c|c|c|c|}
\hline Squint & Defective eye & Nystagmus & Ptosis & $\begin{array}{l}\text { Abnormal } \\
\text { pupils }\end{array}$ \\
\hline
\end{tabular}

OCULAR HISTORY

Are there any known pre-existing ocular conditions, e.g. cataract, retinopathy, macular degeneration?

\section{COGNITION}

Comment of the patient's cognitive / functional / physical ability, i.e. presence of agnosia, alexia, aphasia, hemiplegia, etc.

MEDICATIONS:

EXPECTED DATE OF DISCHARGE (IF IN-PATIENT):

SIGNED:

PRINT NAME:
DATE:

DESIGNATION:

Figure 1 Standardised screening form used by all participating recruitment centres. This doubled as the referral form when completed.

involve all stroke survivors, as provision of screening and assessment for this population was beyond the scope of this study. Standardised screening/referral (Figure 1) and investigation forms are used in each recruiting centre, which identifies known pre-existent ocular pathology, symptoms and signs, investigation of visual field, ocular motility and perceptual aspects, and stroke demographics.

All centres had a linked stroke specialist Orthoptist, who communicated directly with the stroke multidisciplinary team. The stroke team was instructed to complete as much of the screening form as possible, including patient-reported visual symptoms and objective documentation of ocular signs. Where unsure, they indicated it as 'suspected visual difficulty'. All forms were subsequently sent to the Orthoptist and all referred patients were offered appointments for visual assessment by the Orthoptist.

A 'gold standard' assessment was made by the Orthoptist when evaluating the patient's visual status. Visual fields were assessed by confrontation if the patient was seen at the ward or at the rehabilitation unit. When seen in clinic, quantitative measures of visual field were undertaken by Humphrey automated perimeter (Humphrey Instruments, Dublin, CA, USA) and 
Goldmann manual perimeter (Haag Streit, Koeniz, Switzerland). Assessment of ocular alignment and motility consisted of cover test, evaluation of saccadic, smooth pursuit and vergence eye movements, retinal correspondence (Bagolini glasses), fusional vergence (20D or fusional range), stereopsis (Frisby near test), prism cover test, and lid and pupil functions (equipment available from Haag Streit, Harlow, UK). Perceptual deficits were recorded after questioning of patient and/ or carers and relatives. Inattention was assessed by means of a combination of assessments, including line bisection, Albert's test, cancellation tests, and memory tests using verbal description and drawing. Visual acuity was assessed at near and distance fixation with Snellen or logMAR acuity tests (Haag Streit, UK). Stroke details were recorded from patient notes accounting for stroke laterality, type, and area involved. Ocular treatment details were recorded along with outcome.

Statistical analysis was undertaken using the SPSS version 15 software (IBM Corporation, New York, NY, USA). Sensitivity and specificity were calculated based on true and false positive scores. Kappa evaluation of agreement was undertaken and assessed as low, medium, or high agreement between variables.

\section{Results}

A total of 1222 patients (all stroke survivors) were referred with visual problems or suspected visual difficulty to the Orthoptists for assessment. A total of 423 patients were excluded from the study, the latter mainly because of inability to provide informed, written consent (as required of the ethical approval for this study) (Table 1). It was not possible to obtain full visual information on these excluded patients.

In total, 799 stroke survivors were recruited to the study between May 2006 and March 2009. The referral forms for all cases were reviewed. Referring staff comprised the stroke multidisciplinary team of stroke physicians, nursing staff, occupational therapists, physiotherapists, and speech and language therapists. Referrals were made pre-dominantly by occupational therapists and physicians. However, there was no difference in accuracy of referral by any group.

Referring staff could circle specific signs on the screening form, for example, nystagmus, strabismus, ptosis, or specify their own observations such as suspected visual field defect. After screening, this form doubled as the referral form for vision assessment. In $42 \%$ of forms, the signs recorded included suspicion of visual difficulty (23.6\%), abnormal eye movements $(3.2 \%)$, facial nerve palsy $(3.2 \%)$, ptosis $(2.2 \%)$, strabismus $(1.6 \%)$, nystagmus $(1.2 \%)$, abnormal head posture $(0.5 \%)$, visual inattention $(0.5 \%)$, and pupil abnormality $(0.1 \%)$. A combination of these signs was recorded in $6 \%$ of these forms (Figure 2). No signs were recorded on $58 \%$ of these forms. Symptoms were noted in $87 \%$ of patients, (Figure 3 ) and were mainly related to

Table 1 Reasons for exclusion

\begin{tabular}{lccccccc}
\hline $\begin{array}{l}\text { Unable to } \\
\text { consent }\end{array}$ & $\begin{array}{c}\text { Discharged } \\
\text { without assessment }\end{array}$ & $\begin{array}{c}\text { Transient } \\
\text { ischaemic attack }\end{array}$ & $\begin{array}{c}\text { Unwilling } \\
\text { to consent }\end{array}$ & $\begin{array}{c}\text { Not available } \\
\text { for assessment }\end{array}$ & $\begin{array}{c}\text { Died } \\
\text { Other }\end{array}$ & $\begin{array}{c}\text { Failed to } \\
\text { pathology }\end{array}$ & attend assessment \\
\hline 225 & 52 & 44 & 34 & 28 & 26 & 10 & 4 \\
\hline
\end{tabular}

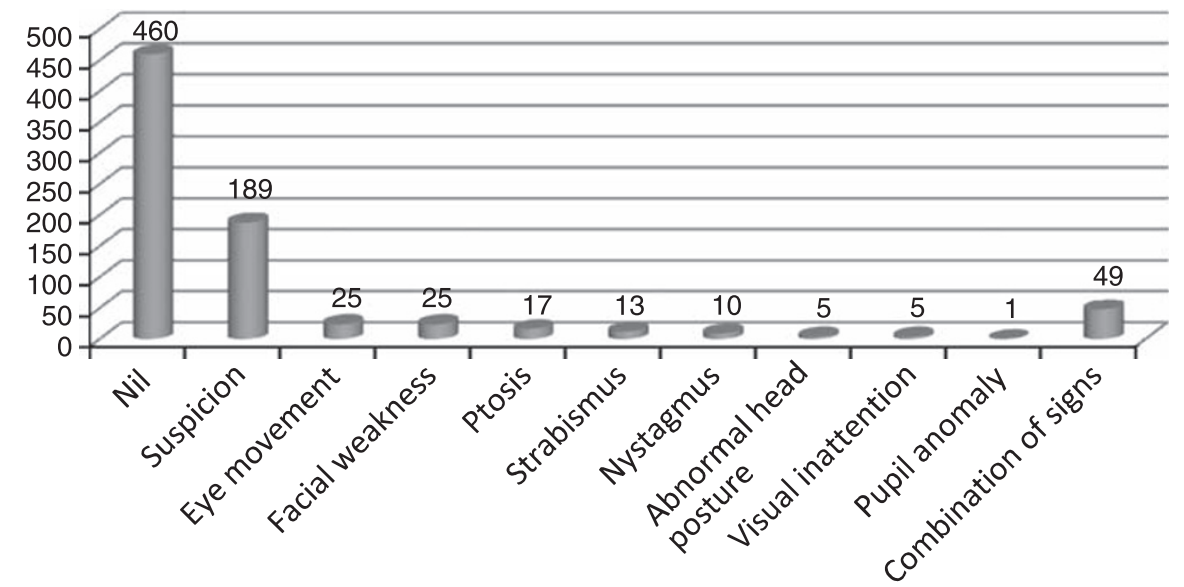

Figure 2 The $x$ axis depicts the visual signs recorded by referring staff. The $y$ axis depicts the numbers of patients. A total of 460 patients $(58 \%)$ had no visual signs recorded by the referring staff. 


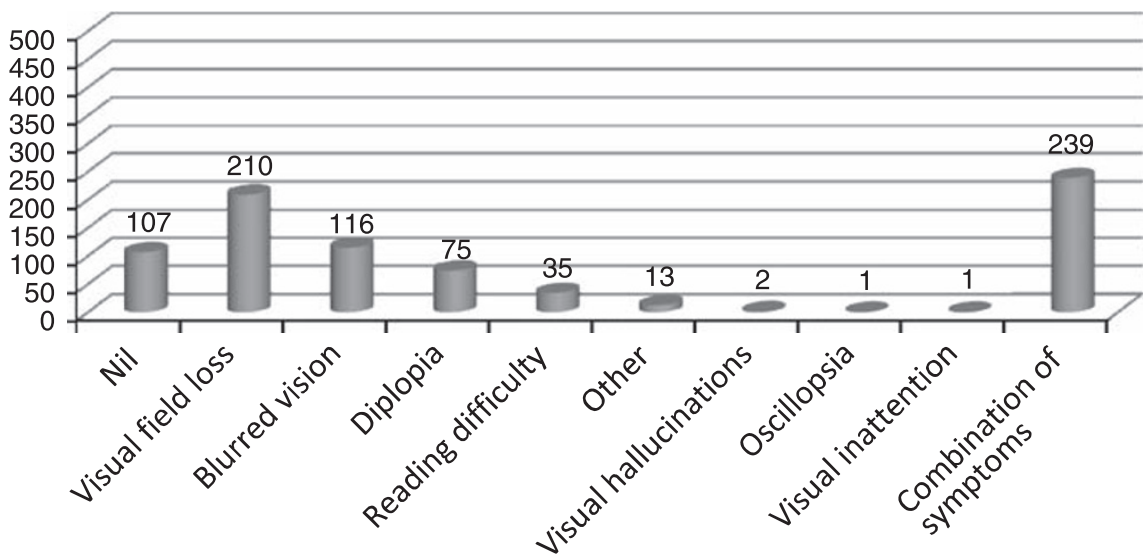

Figure 3 The $x$ axis depicts the visual symptoms recorded by referring staff. The $y$ axis depicts the number of patients. A total of 107 patients $(13 \%)$ had no visual symptoms recorded by the referring staff.

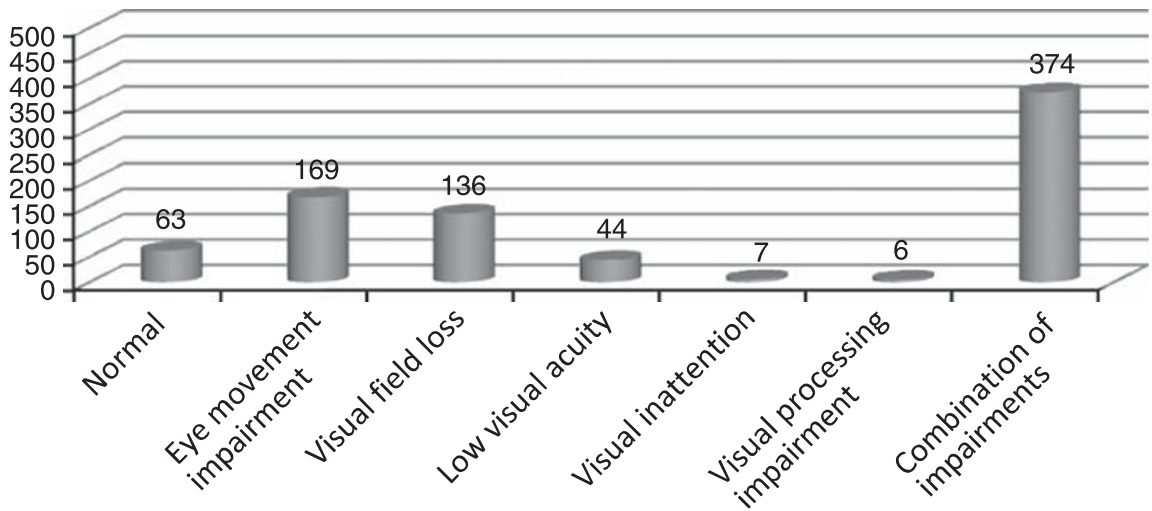

Figure 4 The $x$ axis depicts the visual diagnosis made at eye examination. The $y$ axis depicts the number of patients. A total of 63 patients $(8 \%)$ had no visual anomalies detected when examined by the eye care team.

loss of visual field (26.3\%), blurred vision (14.5\%), double vision $(9.4 \%)$, or a combination of various symptoms (29.9\%). Approximately, $6 \%$ of patients were referred without any identifiable signs or symptoms. Referring staff also reported presence of communication difficulties. Receptive and/or expressive aphasia was documented in 188 patients $(23.5 \%)$.

The first vision assessment was undertaken at a median of 19 days (range 0-2543 days) after the onset of stroke. Three patients (assessment outliers) were referred many years after the onset of their stroke, but were seen for eye assessment within 1 month of receipt of their referral. At vision assessment, a number of ocular diagnoses were made (Figure 4). Normal ocular findings were found in $8 \%$, with $92 \%$ having a confirmed visual impairment of varying type. Abnormal eye movements were found in $21 \%$, visual field loss in $17 \%$, low visual acuity in 5.5\%, visual inattention in $1 \%$, and other visual perceptual abnormalities in $1 \%$.
Combinations of these diagnoses were found in the remaining patients $(46.5 \%)$.

Overall, sensitivity and specificity were calculated for positive and negative referrals (on the basis of recorded signs) $v s$ a positive or negative ocular diagnosis.

Sensitivity value was 0.42 with specificity value of 0.52 . A positive predictive value was calculated at $91 \%$. A Kappa evaluation of agreement was made between the referral accuracy and the diagnosis of visual impairment. This was calculated at 0.428 (SE 0.017: 95\% confidence interval of $-0.048,0.019)$.

Individual signs (excluding suspicion or multiple observations) were considered for their accuracy of detection vs confirmed ocular diagnosis (Table 2). Ptosis was correctly identified in $94 \%$ of patients, strabismus in $92 \%$, nystagmus in $90 \%$, abnormal eye movements in $60 \%$, visual inattention in $60 \%$ and facial nerve palsy in $44 \%$. Abnormal head posture was identified correctly in all cases in which it was noted (100\%). 
Table 2 Accuracy of sign recognition

\begin{tabular}{|c|c|c|c|c|c|c|c|c|}
\hline & Ptosis & Strabismus & Nystagmus & $\begin{array}{c}\text { Abnormal } \\
\text { eye movements }\end{array}$ & $\begin{array}{c}\text { Visual } \\
\text { inattention }\end{array}$ & $\begin{array}{c}\text { Facial } \\
\text { weakness }\end{array}$ & $\begin{array}{c}\text { Abnormal } \\
\text { head posture }\end{array}$ & $\begin{array}{c}\text { Pupil } \\
\text { anomalies }\end{array}$ \\
\hline Total number noted by referrer & 17 & 13 & 10 & 25 & 5 & 25 & 5 & 1 \\
\hline Confirmed on assessment & 16 & 12 & 9 & 15 & 3 & 11 & 5 & 0 \\
\hline$\%$ Accuracy & 94 & 92 & 90 & 60 & 60 & 44 & 100 & 0 \\
\hline
\end{tabular}

Pupil abnormalities were not identified correctly in any case in which it was reported by the referring staff $(0 \%)$.

\section{Discussion}

Approximately, 92\% of all patients referred had a confirmed diagnosis of visual impairment of varying type. Thus, there was a high positive predictive value for referrals demonstrating that consideration of both visual signs and symptoms on the screening form by the stroke multidisciplinary team resulted in appropriate referrals.

It is important that stroke survivors, who have visual difficulty following stroke, are referred for appropriate ocular examination. Many individuals have low vision, ocular alignment or movement problems, visual field loss, visual inattention, and other processing abnormalities following their stroke. ${ }^{1,2,8}$ There are various treatment options for these visual problems, which can have a positive beneficial effect in terms of their vision but also in their general rehabilitation. ${ }^{1,2,9}$ Additionally, many individuals have pre-existent ocular disease such as glaucoma, age-related macular degeneration, and cataract. ${ }^{1,8}$ Ensuring that the correct glasses prescription is available can significantly aid central vision and any treatment regime for coexistent ocular disease must be continued during any inpatient stay for stroke survivors as well as following their discharge. ${ }^{1,2}$

For the above reasons, it is important that patients with visual impairment are identified by the stroke multidisciplinary team and appropriate onward referral made for vision assessment. We sought in this study to evaluate the referral forms, from a large observational study, made by the stroke team, to evaluate the accuracy of the referral information. The study target population was those thought to have a visual problem, and thus referred. Thus, we cannot speculate on patients who may have had visual impairment that was not detected or suspected by the referring staff and this remains an unknown population.

Despite the finding of a high positive predictive value for referrals of suspected visual difficulty, when evaluating the information on the referral form in relation to what was observed by the multidisciplinary team, and thus contributed to their reason for referral, only $42 \%$ of staff reported that ocular signs were evident. Many staff specified 'suspicion' of visual difficulty as a reason for referral. Many types of visual impairment are not visible to an observer such as visual field loss. However, indicators as to their presence should be sought such as visual symptoms, unusual movements of the head to compensate, ignoring one side or closing one eye because of double vision. Where staff were suspicious of a particular defect, for example, visual field defect, this was written on the form. However, no additional information was written on the form when referring staff were unsure of what visual sign might be present. When cross-evaluating the final ocular diagnosis vs the referring signs for accuracy of the visual impairment detected, a sensitivity and specificity value of 0.42 and 0.52 , respectively, were calculated. Kappa analysis of agreement was 0.428 , showing moderate agreement.

Frequently, the identification of ocular signs on assessment by the multidisciplinary team was not consistent with the final ocular diagnosis. This finding has been found in an unpublished audit of stroke referrals for vision assessment, in which $56 \%$ of visual diagnoses made before formal eye assessment were incorrect, with an amended diagnoses being made once visual assessment was undertaken by the Orthoptic and Ophthalmic team. ${ }^{10}$ Accuracy of detection of ocular signs by non-eye health professionals has been reported for referrals with diabetic retinopathy by primary care physicians. ${ }^{11}$ Improvement in referral accuracy was noted and it was felt that education may significantly improve detection rates and appropriate referrals. Indeed, accuracy of diagnosis of stroke has been shown to be poor in non-stroke specialist/neurology physicians. $^{12,13}$

We looked specifically at the type of sign and whether this had a bearing on accuracy. Detection of abnormal head posture, ptosis, strabismus and nystagmus, all had high accuracy between 90 and 100\%, when they were detected although many of these signs also went undetected. Pupil abnormalities were not detected accurately in any case. Thus, when specific ocular signs that are easily 'visible' are identified by the multidisciplinary team, these reports are generally accurate. 
One question relates to the $58 \%$ of referrals being made despite the absence of detection of ocular signs as a reason for referral. The referrals from the group without identified signs were based on the presence of visual symptoms and/or a suspicion of visual difficulty because of altered patient behaviour. Visual symptoms accounted for most reasons for referral in $87 \%$. Only $6 \%$ of referral forms did not identify any visible signs or reported symptoms. This clearly demonstrates that observations of patient behaviour and reports of ocular symptoms are a very important part of screening in the detection of presence of actual visual impairment. The results, thus also raise concerns for patients who are unable to communicate their visual symptoms because of cognitive impairment or aphasia; $23.5 \%$ of patients in this study were documented as having communication difficulties, but it is unknown how many patients from the non-referred stroke population with communication difficulties may have had visual problems.

One potential confounding factor in this study is that the units involved in this study already had a designated specialist Orthoptist liaison in the stroke team. This may have led to a heightened awareness among the multidisciplinary team of the potential for visual impairment among stroke survivors. If more aware of the potential, staff may be more likely to question patients about their vision, thus increasing the detection or suspicion of visual impairment. This form of health promotion is important. Education of referring practitioners has been identified in other areas as being important in the improvement of the accuracy of detection and appropriate referrals. ${ }^{11,14}$ However, where specific liaison with eye services does not exist, this heightened awareness also does not exist. Hence, the detection in such units may be much lower. A prospective trial would be required to substantiate this hypothesis, comparing detection and visual impairment rates across units with and without specialist eye care involvement.

It is known that many stroke units do not have an Orthoptic input to their unit on a formal basis, a number provides only basic qualitative visual assessment, and some have no provision for visual assessment. ${ }^{5}$ Thus, this raises considerable concern for the referral process of patients with visual problems in that it is likely that many remain unidentified or undiagnosed and without referral to appropriate eye care services. A strong recommendation of this study is that every stroke unit implements a visual screening protocol (as a minimum) to consider factors of visual acuity, visual field, eye movement, and vision perception with a clear care pathway for appropriate referral of these patients to eye care services. ${ }^{15}$ It is apparent that a considerable impact occurs to quality of life and activities of daily living, ${ }^{1-4}$ and that early referral is warranted for provision of vision assessment and rehabilitation. ${ }^{15,16}$

In conclusion, accuracy of referrals by the stroke multidisciplinary team when requesting ocular examination has a low sensitivity and specificity when evaluating the identification of presence or absence of ocular signs. Where ocular signs are identified, these are mostly accurately reported. Approximately, 58\% of referrals had no signs detected but were referred on the basis of reported symptoms or suspicion of the patient's behaviour, and knowing that a specialist Orthoptist stroke service was available within the team. Thus, a high positive predictive value was achieved. The results raise concerns for patients who are unable to communicate their visual symptoms and for patients who are in units without specified eye care liaison. Education to promote such a level of knowledge and heightened awareness of visual impairment may improve referrals in which specific links are not available in other stroke units. In addition, vision-screening protocols are required to identify potential visual deficits in stroke survivors with clear care pathways for their onward referral to eye services.

\section{Summary}

\section{What was known before}

- Many stroke survivors have low vision, ocular alignment or movement problems, visual field loss, visual inattention, and other processing abnormalities following stroke.

- Visual rehabilitation has a positive beneficial effect to general rehabilitation in addition to vision.

\section{What this study adds}

- MDT has low sensitivity and specificity when evaluating presence of ocular signs solely. Most MDT eye referrals are based on presence of visual symptoms.

- Concerns exist for patients unable to communicate their visual symptoms. Health education and promotion required to heighten awareness of visual impairment.

\section{Conflict of interest}

The authors declare no conflict of interest.

\section{Acknowledgements}

We thank Alison Price, Birmingham: Sandwell and West Birmingham NHS Trust and Linda Walker, Burnley: East Lancashire Hospitals NHS Trust.

\section{References}

1 Rowe FJ, VIS group. Visual impairment following stroke. Do stroke patients require vision assessment? Age and Ageing 2009; 38: 188-193. 
2 Jones SA, Shinton RA. Improving outcome in stroke patients with visual problems. Age Ageing 2006; 35: 560-565.

3 MacIntosh C. Stroke re-visited: visual problems following stroke and their effect on rehabilitation. Br Orthopt J 2003; 60: 10-14.

4 Ramrattan RS, Wolfs RC, Panda-Jonas S, Jonas JB, Bakker D, Pols HA et al. Prevalence and causes of visual field loss in the elderly and associations with impairment in daily functioning: the Rotterdam Study. Arch Ophthalmol 2001; 119: 1788-1794.

5 Rowe FJ. Who sees visual impairment following stroke? Strabismus 2009; 17: 37-40.

6 Department of Health. National Stroke Strategy. DH: London, 2007.

7 Royal College of Physicians Intercollegiate Stroke Working Party. National Clinical Guidelines for Stroke, 3rd edn. Royal College of Physicians: London, 2008.

8 Lotery AJ, Wiggam MI, Jackson AJ, Silvestri G, Refson K, Fullerton KJ et al. Correctable visual impairment in stroke rehabilitation patients. Age and Ageing 2000; 29 221-222.

\section{Appendix}

\section{VIS Group:}

David Wright, Altnagelvin: Western Health and Social Services Trust; Darren Brand, Ayr: NHS Ayrshire and Arran; Carole Jackson, Bath: Royal United Hospitals Bath NHS Trust; Shirley Harrison, Bury: Bury PCT; Carla Eccleston, Derby: Derby Hospitals NHS Trust; Tallat Maan, Durham: County Durham and Darlington NHS Foundation Trust; Claire Scott, Ipswich: Ipswich Hospital NHS Trust; Sarah Peel, St Helier: Jersey; Linda Vogwell, Gloucester: Gloucestershire Hospitals NHS Foundation
9 Freeman CF, Rudge NB. Cerebrovascular accident and the Orthoptist. Br Orthopt J 1988; 45: 8-18.

10 Brand D. Parallel vision. British and Irish Orthoptic Society 2009; 126 (6): 1.

11 Awh CC, Cupples HP, Javitt JC. Improved detection and referral of patients with diabetic retinopathy by primary care physicians. Arch Intern Med 1991; 151: 1405-1408.

12 Moeller JJ, Kurniawan J, Gubitz GJ, Ross JA, Bhan V. Diagnostic accuracy of neurological problems in the emergency department. Can J Neurol Sci 2008; 35: 335-341.

13 Fischer CE, Barnung S, Nielsen SL, Rasmussen LS. Prehospital identification of stroke-room for improvement. Eur J Neurol 2008; 15: 792-796.

14 Fink A, Wright L, Wormald R. Detection and prevention of treatable visual failure in general practice: room for improvement? Br J Gen Pract 1994; 44: 587-589.

15 Ijaola FO, Kausar SA. Visual impairment following stroke: do stroke patients require vision assessment? Letter to editor. Age and Ageing 2009; 38: 629-630.

16 Rowe FJ, VIS group. Visual impairment following stroke: do stroke patients require vision assessment? Letter to editor. Age and Ageing 2009; 38: 629-630.

Trust; Leonie Robson, Lincoln: United Lincolnshire Hospitals NHS Trust; Nicola Akerman, Nottingham: University Hospital NHS Trust; Caroline Dodridge, Oxford: Oxford Radcliffe Hospitals NHS Trust; Claire Howard, Salford: Salford Primary Care Trust; Tracey Shipman, Sheffield: Sheffield Teaching Hospitals Foundation Trust; Una Sperring, Swindon: Swindon and Marlborough NHS Trust; Sue Yarde, Taunton: Taunton and Somerset NHS Trust; Sonia MacDiarmid, Wigan: Wrightington, Wigan, and Leigh NHS Trust; Cicely Freeman, Worcester: Worcestershire Acute Hospitals NHS Trust. 